\title{
Efferocytosis in Retinoblastoma
}

\author{
Dipankar Das*, Harsha Bhattacharjee, Kasturi Bhattacharjee, Prerana S. Tahiliani, \\ Pankaj Bhattacharyya, Manabjyoti Barman, Sumita Sarma Barthakur, Panna Deka, \\ Apurba Deka, Rajashree Paul
}

Sri Sankaradeva Nethralaya, Guwahati, India.

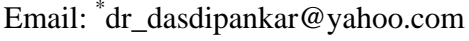

Received October $25^{\text {th }}, 2013$; revised November $17^{\text {th }}, 2013$; accepted November $24^{\text {th }}, 2013$

Copyright (c) 2013 Dipankar Das et al. This is an open access article distributed under the Creative Commons Attribution License, which permits unrestricted use, distribution, and reproduction in any medium, provided the original work is properly cited.

\begin{abstract}
Retinoblastoma has varied presentations. As age advances in retinoblastoma, the undifferentiated tumour is the common appearance. Apoptosis in retinoblastoma in an untreated case can give us a clue about the tumour biology. Efferocytosis in intraocular tumour is a new concept which was seen in an enucleated eyeball specimen in a 4-year-old boy. Effective efferocytosis can be conceptualized for new pharmacodynamics application for anti-cancer drugs. We present a rare case of retinoblastoma with efferocytosis in retinal pigment epithelium and interesting apoptotic changes.
\end{abstract}

Keywords: Retinoblastoma; Apoptosis; Efferocytosis; Vesicles; Undifferentiated

\section{Introduction}

Retinoblastoma is the common intraocular tumour in children [1]. The survival of children with retinoblastoma, if diagnosed early, has gone up to 95\% in advanced centers $[1,2]$. Apoptosis and programmed cell death in these tumours are important observations to understand the tumour biology [2-4]. It is always a challenge to explore apoptosis for understanding the cancer treatment [2-7].

Interesting pathological observations of apoptotic changes in a unilateral, undifferentiated and untreated case of retinoblastoma were seen where nuclear fragmentation and apoptotic vesicle arrangement were realized and macrophages in the process showed their phagocytic actions on the apoptotic vesicles. A new phenomenon of cell biology called "efferocytosis" was documented for the first time in the retinoblastoma.

\section{Case Report}

A 4 years old male child reported to a tertiary institute of northeast India with a complaint of white pupillary reflex in the right eye (OD) noted by his parents for past 15 days. On examination, his vision in the OD was perception of light positive and 6/6, N6 in the left eye (OS). Anterior segment findings were normal in OD and on dilated fundus examination of the $\mathrm{OD}$, a portion of the

\footnotetext{
${ }^{*}$ Corresponding author.
}

white mass with calcified extension was seen with associated retinal detachment Figure 1. The case was clinically diagnosed Group E retinoblastoma of the OD. B scan ultrasound of the OD showed a superior temporal mass with adjoining calcification and calcified area had high reflectivity on A-scan. There was no extra ocular extension noted on the ultrasound. Computed tomography scans of brain and orbit revealed retinoblastoma mass confined to the right eye ball without any obvious extra ocular extension. Enucleation of the OD with silicon ball implantation was done and examination under anesthesia was carried out in the OS in the same sitting. The enucleated specimen was sent to the ocular pathology laboratory of the institution where it was fixed overnight.

\subsection{Gross Description}

The enucleated eyeball measured anterior-posteriorly $22.07 \mathrm{~mm}$, horizontally $23.18 \mathrm{~mm}$, vertically $22.63 \mathrm{~mm}$; cornea measured horizontally $11.52 \mathrm{~mm}$, vertically 10.83 $\mathrm{mm}$; pupil measured $5.17 \mathrm{~mm}$ in diameter. Optic nerve measured $10.10 \mathrm{~mm}$ in length and $3.25 \mathrm{~mm}$ in diameter along with meninges. White pupillary reflex was seen. White calcified area measuring $12.37 \mathrm{~mm}$ by maximum height $9.14 \mathrm{~mm}$ was seen separately from freshly looking main tumour mass measuring $18.21 \mathrm{~mm} \times 11.53 \mathrm{~mm}$ Figure 2. Lens was pushed forward. There was retinal 


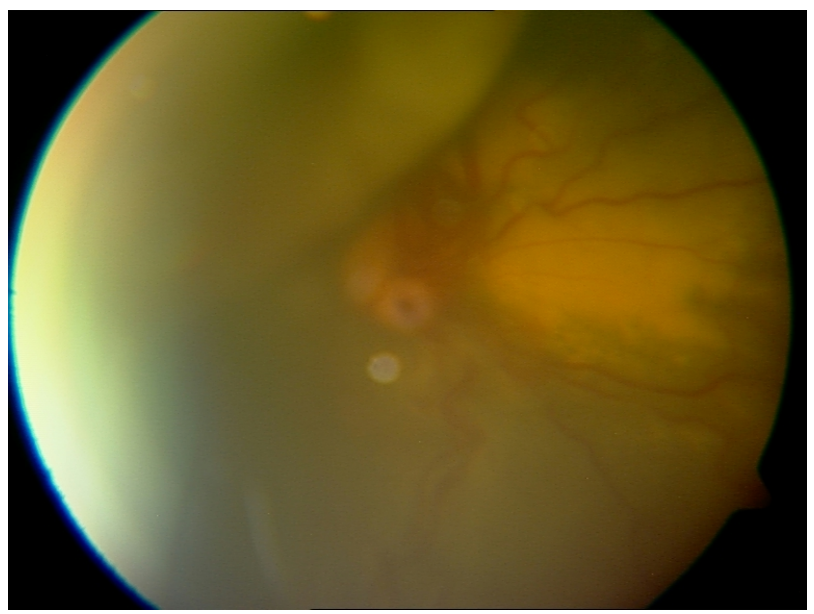

Figure 1. Fundus examination of the right eye showing a portion of the white mass with calcified extension and associated retinal detachment.

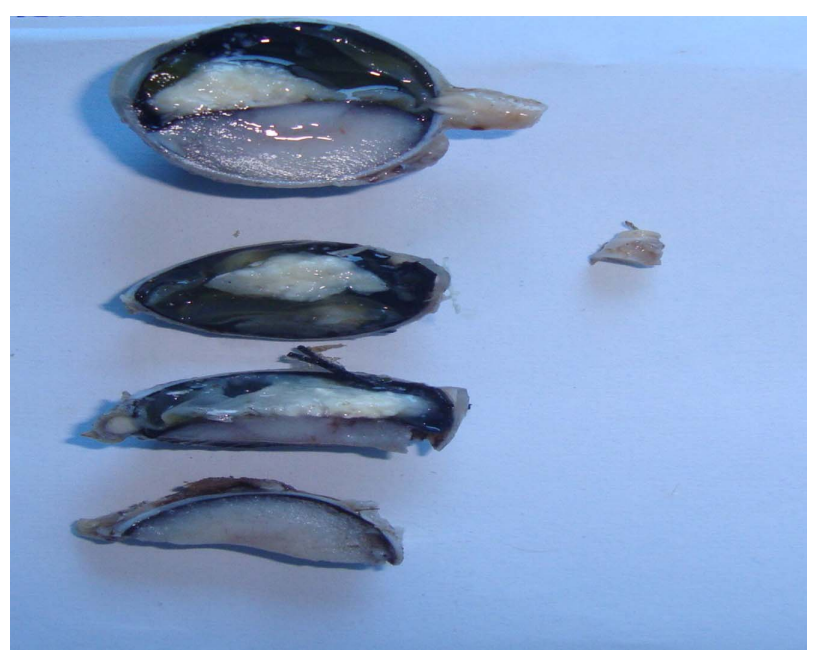

Figure 2. Showing white calcified area seen separately from freshly looking main tumour mass and the lateral calottes with cut section of distal end of optic nerve.

detachment seen superiorly. Sclera seemed to be normal. By bread loaf technique, lateral calottes were cut and submitted. Transillumination defect was seen in the vitreous cavity. Eyeball was sectioned vertically. Cut end of the optic nerve was submitted separately and examined.

\subsection{Microscopic Description}

The enucleated eyeball showed normal corneal layers. A focus of tumour was seen behind the iris and in one of the detached angles Figure 3. Portion of lens was normal. A basophilic tumour mass in the vitreous cavity was seen which was endophytic in nature with necrosis and calcification. The tumour was undifferentiated with intra scleral tumour invasion was seen focally Figure 4. There were unusual pigmented macrophages seen in the main tumour mass and also in the area of scleral invasion Fig- ure 5. The pigmented macrophages were seen tracking from the main tumour mass to the scleral invasion Figure 6. Cells contained undifferentiated, pleomorphic and basophilic retinoblastoma cells and vesicular (apoptotic) bodies Figure 7. The macrophages showed engulfment of vesicular bodies Figure 8. Some cells showed apoptosis in the tumour mass where fragmentation of nuclear component were seen along with vesicle formation Figure 9. Retinal pigment epithelium (RPE) showed the phenomenon of efferocytosis where macrophages were seen engulfing apoptotic vesicles Figure 10. The vesicles (efferosome) were in different shapes and sizes noted in the RPE Figure 11. There was no extra scleral involvement and no tumour invasion was seen in the attached optic nerve. Lateral callotes showed full thickness choroidal involvement. Portion of the cut end of the optic nerve did not show tumour invasion.

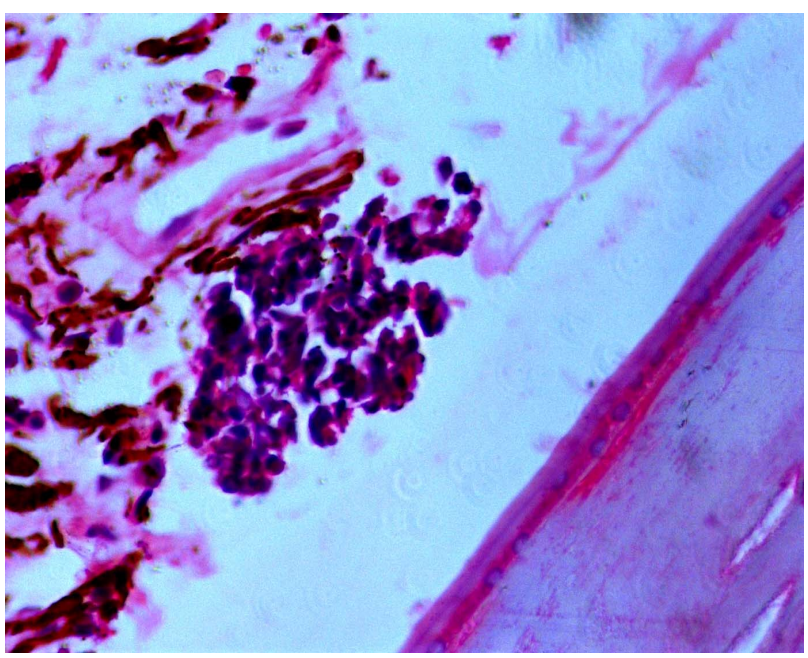

Figure 3. $(\mathrm{HE}, \times 200)$ Showing a focus of tumour behind the iris and in one of the detached angles.

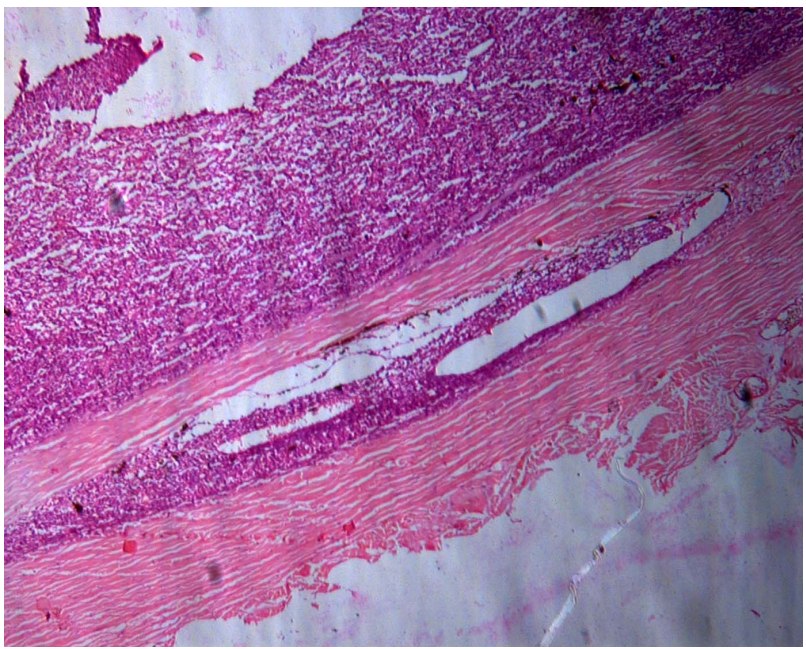

Figure 4. $(\mathrm{HE}, \times 100)$ Showing focal intra scleral tumour invasion. 


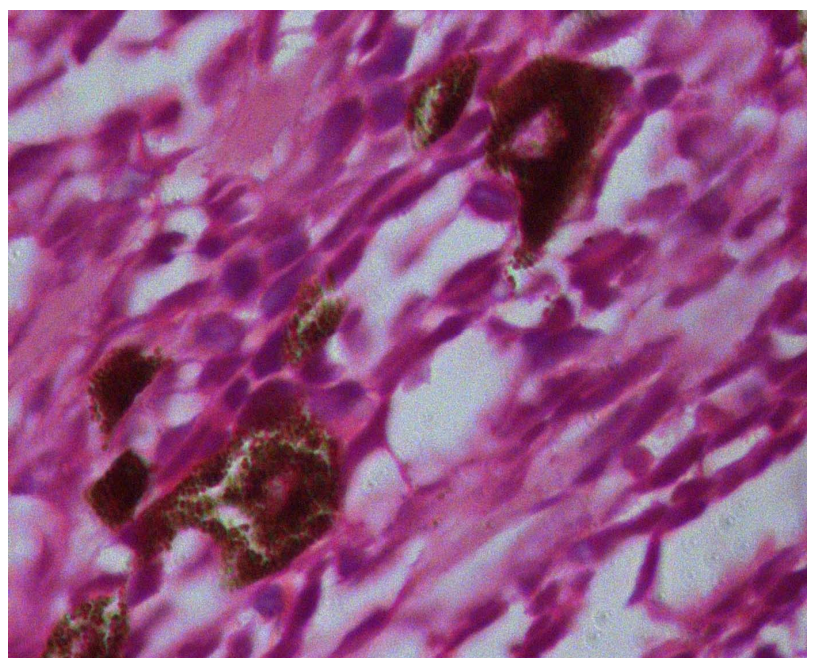

Figure 5. (HE, $\times 400)$ Showing presence of unusual pigmented macrophages in the main tumour mass and also in the area of scleral invasion.

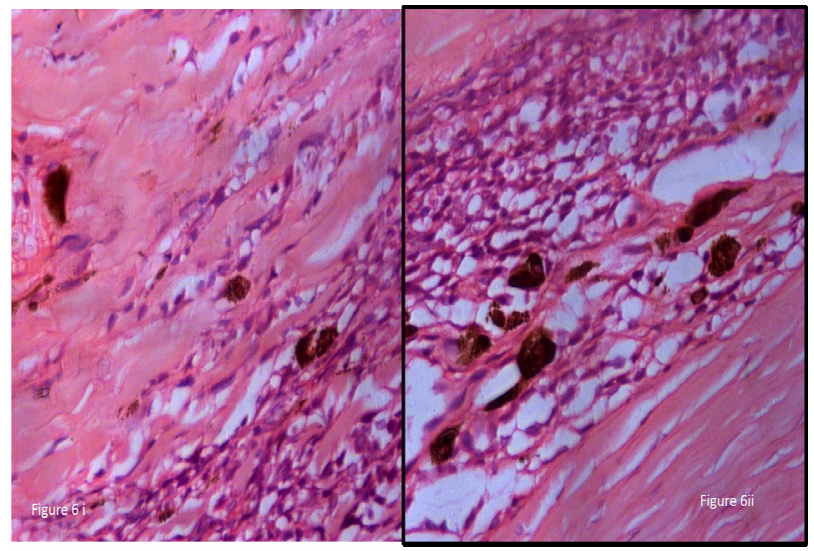

Figure 6. Showing the pigmented macrophages tracking from the main tumour mass to the scleral invasion.

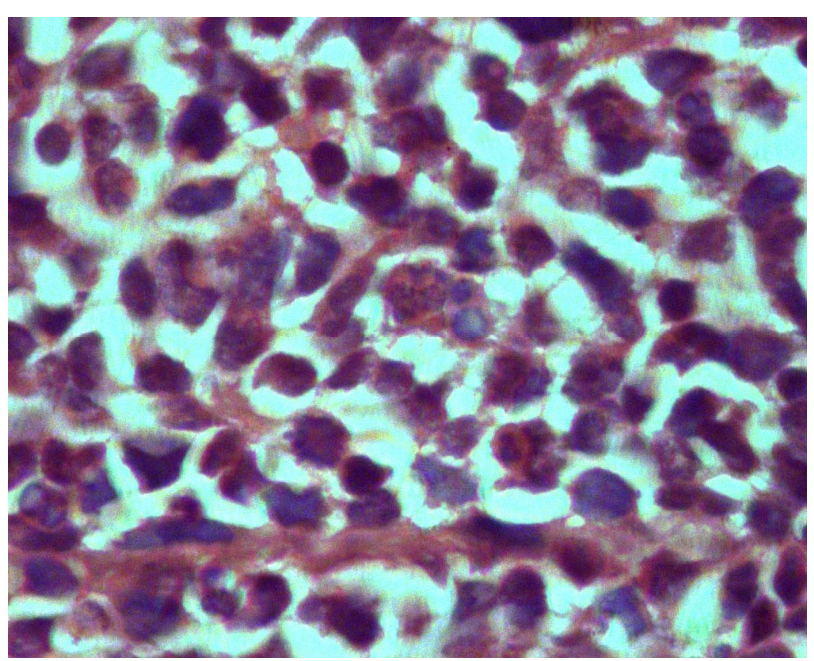

Figure 7. (HE, $\times 400)$ Showing undifferentiated, pleomorphic and basophilic retinoblastoma cells and vesicular (apoptotic) bodies.

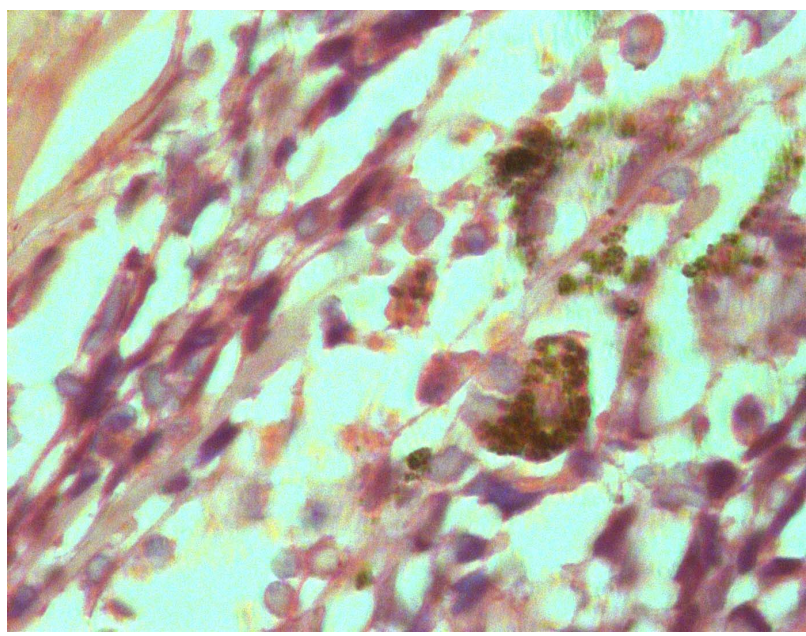

Figure 8. $(\mathrm{HE}, \times 400)$ Showing the macrophages in the process of engulfing the vesicular bodies.

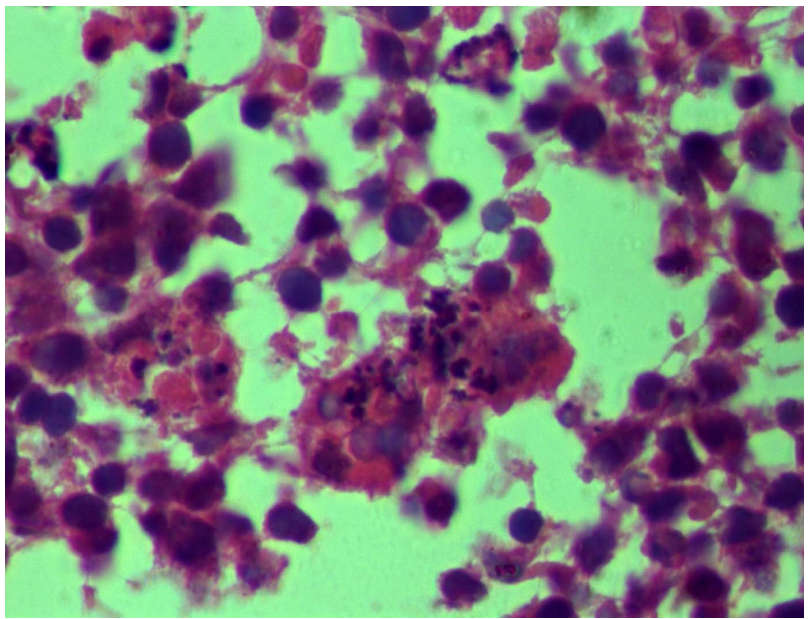

Figure 9. (HE, $\times 400)$ Showing some cells in apoptosis in the tumour mass where fragmentation of nuclear components seen along with vesicle formation.

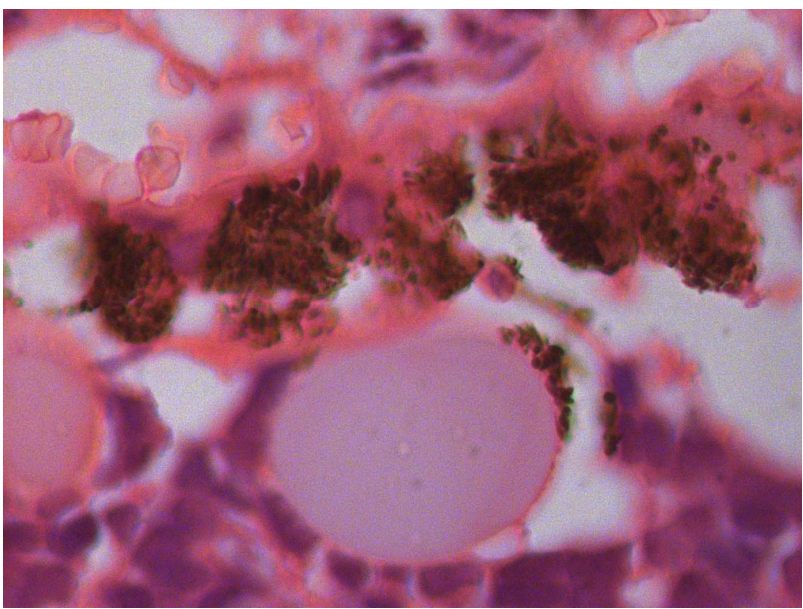

Figure 10. (HE, $\times 400)$ Showing retinal pigment epithelium (RPE) and the phenomenon of efferocytosis where macrophages were seen engulfing apoptotic vesicles. 


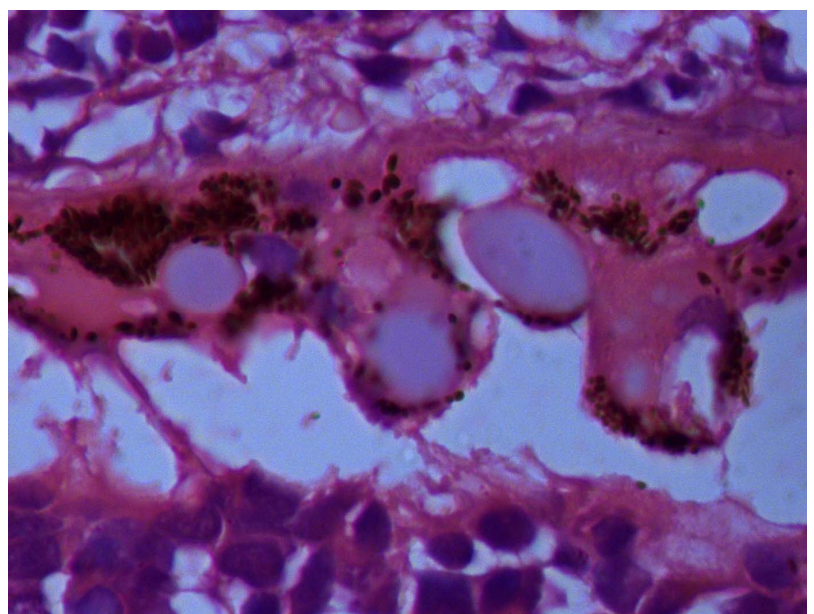

Figure 11. (HE, $\times 400)$ Showing vesicles (efferosome) in different shapes and sizes noted in the RPE.

\section{Discussion}

Efferocytosis is derived from the Latin word "effere" for "to bury", and it is the process by which the dead cells are removed by macrophage phagocytic cells [3-5]. During efferocytosis, the cell membranes of phagocytic cell engulf the apoptotic cells forming a large fluid filled vesicle containing the dead cells [3-6]. The ingested vesicle is called efferosome and this process is similar to macropinocytosis [3,4]. Documentation of efferocytosis in retinal pigment epithelial cells in retinoblastoma specimen of human eye was shown for the first time in the scientific literature. Apoptosis or programmed cell death is orderly and genetically controlled form of cell death which can be seen in the tumour or when chemotherapy induces such change in the tumour mass [5,6]. In a morphological logic, it differs from necrosis in which cellular shrinkage and chromatin condensation occur, followed by fragmentation of nuclear components and apoptotic vesicle formation which are cleared by phagocytosis without damaging the neighboring tissues $[5,6,8]$. We had seen that in our case where nuclear fragmentation and apoptotic vesicle arrangement and the macrophages in the process engulfed the vesicular bodies, some of the vesicles were also seen in the RPE where they were engulfed by the macrophages and thus effective efferocytosis was recognized. These have major pharmacodynamic applications for the anticancer drugs. The first step of any anticancer drug is the interaction of cellular target [59]. As we have seen in our case, there were two groups of cells in the tumour mass and the portion where it had spread i.e. sclera. One group of cells was typical undifferentiated retinoblastoma cells which were basophilic nucleated cells with scanty cytoplasm and the other group was the vesicular cells. So the targeted chemotherapy needs to focus on the vesicular apoptotic cells which can have local effect and then they will be also phagocytosed by the RPE cells. Thus, the effective drug will be delivered in the tumour mass and then eliminated out as efferosome during the process. In nutshell, cells that undergo apoptosis form the second step where the efficacy of anticancer drugs was mediated by those targeted apoptotic vesicles. In larger study, the extent of expression of various oncogenes such as P-53 [9], bcl-2, bax, c-myc and others and exploring the endogenous factors will benefit the understanding of cancer biology of retinoblastoma. It is always a challenge for pharmacological research to explore apoptosis by modulating the extrinsic and intrinsic regulators in a positive and negative direction in order to improve the efficacy of anticancer treatment.

Our case was a 4-year-old boy with unilateral, undifferentiated retinoblastoma. Clinical picture and gross specimen showed two distinct zones of involvement. One was calcified area and the other was a fresh active greyish coloured mass with retinal detachment. We presumed that patient might have small endophytic lesion earlier which spontaneously regressed and later on, there was recurrence. The biology of such mixed tumour with that situation could also be explained even for the apoptotic change. The patient had no history of previous chemotherapy. Tumour necrosis, calcification and obvious intra-scleral spread might be due to the aggressive nature of recurrence. There was an interesting macrophagic change seen in the sclera where the tumour was invaded. Definite track of macrophagic migration was noted to the sclera from the tumor mass. The pigmented macrophages were larger from the normal pigmented cells seen in and around that area of sclera and the tumour. Whether macrophages traveled from the mass to the invaded tissue before actual basophilic tumor cells migration or macrophages really carried the tumour antibodies or proteins to the distant areas in retinoblastoma will be the subject of interest for the researcher in future.

\section{Acknowledgements}

The authors are indebted to: 1) Sri Kanchi Sankara Health \& Educational Foundation (SKSH\&F); 2) Arjun Mandal, Optometrist, Agartala, Tripura, India; 3) Mr. Tarit Das, FM, Sri Sankaradeva Nethralaya, Guwahati, Assam, India for their help and assistance.

\section{REFERENCES}

[1] C. L. Shields, A. Mashayekhi, H. Demirci, A. T. Meadows and J. A. Shields, "Practical Approach to Management of Retinoblastoma," Archives of Ophthalmology, Vol. 22, No. 5, 2004, pp. 729-735. http://dx.doi.org/10.1001/archopht.122.5.729

[2] R. C. Eagle Jr., "High-Risk Features and Tumour Differentiation in Retinoblastoma: A Retrospective Histopatho- 
logic Study," Archives of Pathology \& Laboratory Medicine, Vol. 133, 2009, pp. 1203-1209.

[3] A. H. Wellie, J. F. R. Kerr and A. R. Currie, "Cell Death: The Significance of Apoptosis," International Review of Cytology, Vol. 68, 1980, pp. 251-306. http://dx.doi.org/10.1016/S0074-7696(08)62312-8

[4] R. S. Scott, E. J. Mcmohan, S. M. Pop, E. A. Reep, R. Caricchio, P. L. Cohen, H. S. Earp and G. K. Matsunshine, "Phagocytosis and Clearance of Apoptotic Cells Is Mediated by MER,” Nature, Vol. 411, No. 6834, 2001, pp. 207-211. http://dx.doi.org/10.1038/35075603

[5] A. H. De Cathelineau and P. H. Henson, "The Final Step in Programmed Cell Death: Phagocytes Carry Apoptotic Cells to the Grave,” Essays in Biochemistry, Vol. 39, 2003, pp. 105-117.

[6] R. W. Vandivier, P. M. Henson and I. S. Douglas, "Burring the Dead: The Impact of Failed Apoptotic Cell Removal (Efferocytosis) on Chronic Inflammatory Lung
Disease,” Chest, Vol. 129, No. 6, 2006, pp. 1673-1682. http://dx.doi.org/10.1378/chest.129.6.1673

[7] S. J. Garalei, K. A. McPhillips, S. C. Frasch, et al., "Cell Surface Calreticulin Initiates Clearance of Viable or Apoptotic Cells through Transactivation of LRP on Phagocyte,” Cell, Vol. 123, No. 2, 2005, pp. 321-334. http://dx.doi.org/10.1016/j.cell.2005.08.032

[8] M. H. Cesan, K. Pegan, A. Spes and B. Turk, "Lysosomal Pathways to Cell Death and Their Therapeutic Application,” Experimental Cell Research, Vol. 318, No. 11, 2012, pp. 1245-1251. http://dx.doi.org/10.1016/j.yexcr.2012.03.005

[9] J. S. Lee, Y. J. Kim, C. L. Kim and G. M. Lee, "Differential Induction of Autophagy in Caspase 3/7 Down Regulating and Bcl-2 Overexpressing Recombinant CHO Cells Subjected to Sodium Butyrate Treatment," Journal of Biotechnology, Vol. 161, No. 1, 2012, pp. 34-41. http://dx.doi.org/10.1016/j.jbiotec.2012.05.011 